\title{
Volatiles from Prunus persica Flowers and Their Correlation with Flower-Visiting Insect Community in Wanbailin Ecological Garden, China
}

\author{
Xiujuan Du ${ }^{1,2^{*}}$, Peter Witzgall2 ${ }^{2}$ Kongming $\mathrm{Wu}^{3}$, Fengming Yan ${ }^{4}$, Chunsen $\mathrm{Ma}^{3}$, Hua Zheng5, \\ Fang Xut ${ }^{6}$, Guoqiang Ji ${ }^{1}$, Xiaohong $\mathrm{Wu}^{1}$
}

${ }^{1}$ Department of Forestry, Shanxi Forestry Vocational Technical College, Taiyuan, China

${ }^{2}$ Department of Plant Protection, Swedish University of Agricultural Sciences, Alnarp, Sweden

${ }^{3}$ Institute of Plant Protection, Chinese Academy of Agricultural Sciences, Beijing, China

${ }^{4}$ College of Plant Protection, Henan Agricultural University, Zhengzhou, China

${ }^{5}$ Research Institute of Resources Insects, Chinese Academy of Forestry, Kunming, China

${ }^{6}$ Public Analysis and Testing Center, Beijing Forestry University, Beijing, China

Email: ${ }^{\star}$ dudragonfly@163.co

How to cite this paper: Du, X.J., Witzgall, P., Wu, K.M., Yan, F.M., Ma, C.S., Zheng, H., Xu, F., Ji, G.Q. and Wu, X.H. (2018) Volatiles from Prunus persica Flowers and Their Correlation with Flower-Visiting Insect Community in Wanbailin Ecological Garden, China. Advances in Entomology, 6, 116-133.

https://doi.org/10.4236/ae.2018.62009

Received: January 12, 2018

Accepted: April 21, 2018

Published: April 24, 2018

Copyright (c) 2018 by authors and Scientific Research Publishing Inc. This work is licensed under the Creative Commons Attribution International License (CC BY 4.0).

http://creativecommons.org/licenses/by/4.0/

\section{(c) (i) Open Access}

\begin{abstract}
Object: To investigate the VOCs from living Prunus persica flowers of different branches and their correlation with floral insects. Special, dominant and 20 VOCs were analysed from living Prunus persica flowers captured by closely and circularly headspace way in adsorbent tubes and ATD-GC/MS on 29 April in Wanbailin Ecological Garden, China. VOCs from high altitude tree are more than low altitude in sunny site, and floral volatile constituents from upslope branches are less than downslope of the same tree on high altitude. Special floral VOCs were alpha-dimethyl-benzenemethanol, isopropyl palmitate, ethylbenzene, p-xylene, acetophenone, 3-ethyl-2-methyl-Heptane on sunny slope, and propylene glycol, decanal, hexadecane on shady slope. Dominant VOCs founded during 8 temporal quanta were toluene, hexane, 2-ethyl-1-hexanol, dodecane, pentadecane. Floral VOCs' number from sunny slope was significantly negative correlated with flower-visiting insect community richness, abundance, diversity on sunny or shady slope, which from shady slope was significantly positive correlated with flower-visiting insect community parameters on both slopes tried dividing insects visiting floral branches from Prunus persica in sunny site with $\mathrm{n} / 2+1$ or $\mathrm{n} / 2-1$, and shady site with $\mathrm{n} / 2$ before sampled volatiles day. On sampled volatiles day, 2-ethyl-1-hexanol from sunny slope was significantly positive correlated with flower-visiting insect community parameters on shady slope $(P<0.001)$. After
\end{abstract}


sampled day, hexanal, toluene, dodecane were significantly negative correlated with SUSR, SUSA, SUSD, and positive with SHSR, SHSD, while tetradecane, pentadecane were positive related to SUSR, SUSA, SUSD with $\mathrm{n} / 2-1$ dividing insects visiting floral branches, but reversely with $\mathrm{n} / 2+1$ dividing insects visiting floral branches. We concluded that special and dominant volatiles applied to lure flower-visiting insects to promote pollination, and odd floral branches should be kept for every tree when pruning the Prunus persica on sunny slope, and even floral branches kept on shady slope.

\section{Keywords}

Prunus persica Flowers, Volatiles, Flower-Visiting Insect, Chemical Response

\section{Introduction}

Many insect groups are important mutualistic partners of plants, mostly due to their roles as pollinators [1] [2]. Volatiles from plant flowers attract nectar-seeking and pollen-collecting pollinators to visit and pollinate by supplying rewards [3] [4] [5] [6]. Meanwhile, volatiles from floral branches prevent flower-robbers from foraging and pollinating [7] [8]. Components from floral scents and herbivore induced flower-volatiles include alcohols, aldehydes, ketones, organic acids, ester, terpenes, nitrogen compounds, hydrocarbons [9] [10] [11] [12], as well as other chemicals varying with plant species and volatile collection types [13]-[19].

Floral scents from 4 cultivated varieties Prunus persica were analysed [20] [21], but the effect of these volatiles on pollinator attraction is unknown. The evolution of floral traits in the context of attracting mutualist or antagonist pollinators may place conflicting selection pressures on plant traits, and the effect of these volatiles on pollinator attraction is indeterminate [22]. Many flowers produce volatiles at times when pollinators are most active; VOCs such as a kind of muurolene are characteristics of the syconium pollen receptive diurnal phase, and may attract the diurnally-active pollinating wasps [23]. In some specialized pollination context, only one or a few specific floral volatiles mediate communication with specific pollinator species, and pollinators are attracted by a blend of widespread volatiles [24]. Specialized relationship between specialized pollinator and host-plant flowers is commonly mediated by specific volatiles [25].

Pollinator visitation is influenced by composition and quantity of flower volatile in long distance, dependent on the pollinator rewards, i.e., pollen and nectar in short distance, and volatile emission by flowers changed upon feeding by herbivores on the leaves when Pieris brassicae caterpillars were allowed to feed only on the leaves of Brassica nigra plants [26]. The volatile composition of floral scents is not static, spatiotemporal variability in the identity and complexity of scent bouquets could provide critical information for the mediation of plant-insect communication because floral visitors can use subtle difference in 
volatiles to make foraging choices [27] [28]. Floral scents have the advantage in that they can supply pollinators a species-specific identity to flowers with their potentially infinite composition and diversity [25] [27]. That Prunus persica fruits need flower-visiting insects, and floral scents play a key role in pollinating. We investigated the floral scent volatile bouquets of Prunus persica, and tested VOCs composition, similarity of different trees, dominant floral volatile dynamic, floral scents correlation with flower-visiting insect community richness, abundance and diversity before, on or after volatiles sampled day with dividing sampled insects visiting floral branches by odd or even method. We report the use of living flowers captured by closely and circularly headspace way in adsorbent tubes and ATD-GC/MS analysed 20 VOCs from Prunus persica flowers, and floral scents correlation with flower-visiting insect richness, abundance and diversity, which are special and dominant floral scents and what the similarity from Prunus persica in different spatial and temporal branches. Are the dominant floral odors temporal dynamic varied differently, and floral odors with difference from temporal trees. We expected that get the special and dominant floral odors from different sampled branches, which led us to hypothesize the potential attractive floral odors that insects locating on trees. Does VOCs correlate with floral insect richness, abundance and diversity, we want to know that the insects visiting odd or even flower branches correlated with floral odor closely, so that definite the branches kept when pruning Prunus persica, and promote harvest to get economic benefit.

\section{Sites and Methods}

Sample sites that the $1280.0 \mathrm{~m}$ altitude of sunny and shady slope habitats in Wan Bailin Ecological Garden, Shanxi Province, China (37 $\left.44^{\prime} 40^{\prime \prime N}, 112^{\circ} 21^{\prime} 53^{\prime \prime E}\right)$, with temperature $9^{\circ} \mathrm{C}-31^{\circ} \mathrm{C}$, humidity $27 \%-48 \%$, precipitation probability $5 \%$ - 90\%, sunshine duration 2 - 12 h, 2 - 3 level wind, UV intensity weak-strong, atmospheric pressure 1002 - 1016 Pa, 05:00-5:52 sunrise, 19:06-19:53 sundown. 186 plants Prunus persica are surrounded by the Sophora japonica in the West, Berberis thunbergii cv. Atropurpurea pruning balls in the South, and Malus micromalus in the Northeast. On shady slope, 169 plants of Prunus persica are surrounded by Armeniaca vulgaris in the West, Salix matsudana in the South, and garden road in the North (Figure 1).

Volatile collections were conducted from 2 trees of Prunus persica on sunny and shady slope on 29 April 2016, respectively. Two fully blooming floral branches of each tree were sampled. Food grade polythene bag $(40 \mathrm{~cm} \times 28 \mathrm{~cm})$ was used to include the branches with inlet and outlet glass tube with absorbent carbasus $(1 \mathrm{~cm} \times 8 \mathrm{~cm})$. QC-1S Atmospheric sampling instrument (Beijing Labor Protection Research Institute) was used to generate the air flow of $1.5 \mathrm{~L} / \mathrm{min}$ filtered with activated carbon. Each collection was done $10 \mathrm{~min}$.

Volatiles analysis were conducted by Turbo Matrix 50 ATD (Automatic thermal desorption) and Clarus GC (Chromatography)/Clarus 600 T MS (Mass 


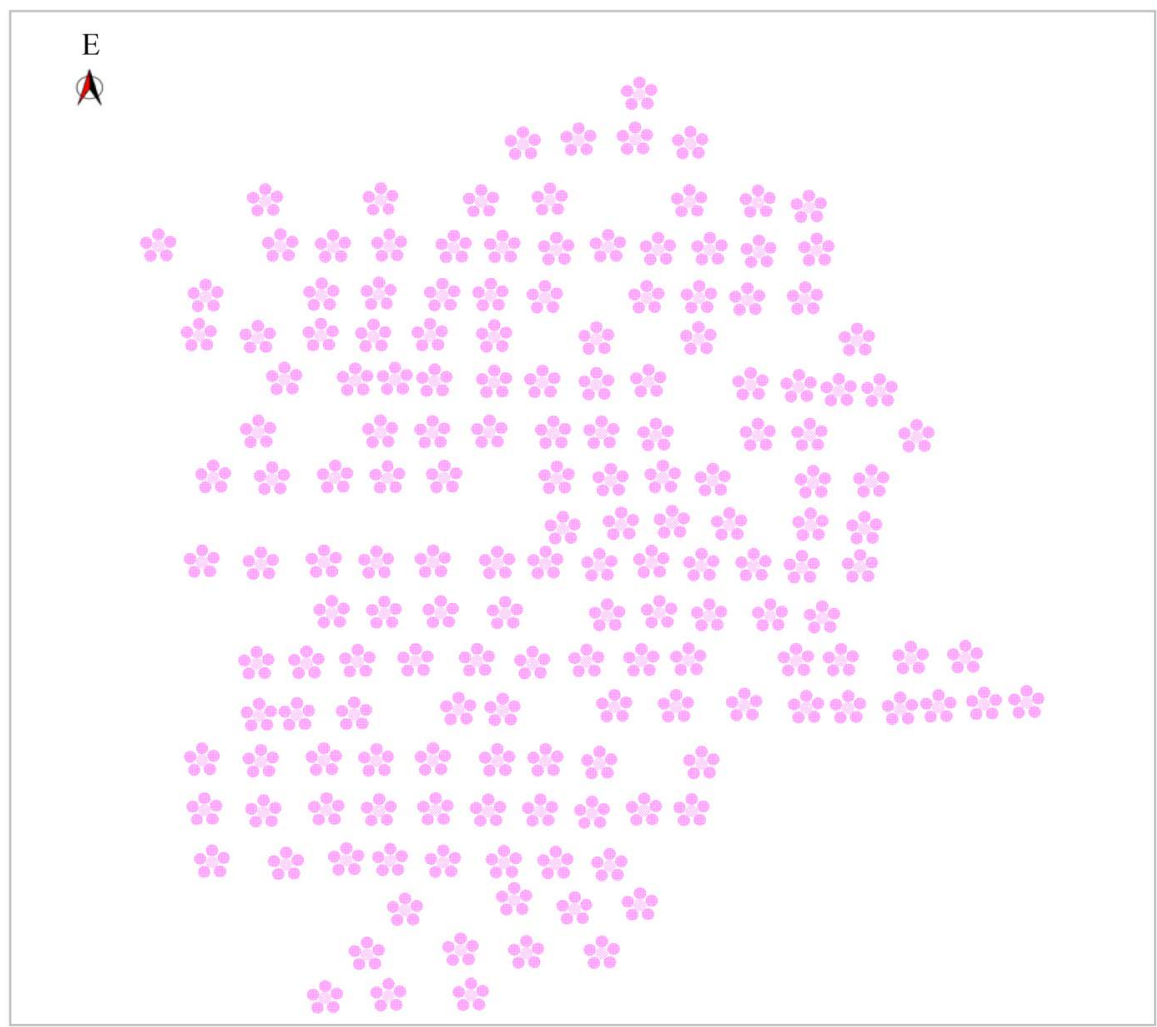

Figure 1. Prunus persica distributed in shady site.

sectrometry) (American Perkin Elmer) with Turb oMass 5.4.2 recognition pattern were used for chemical analysis of collected volatiles. Carrier gas velocity was $2.0 \mathrm{ml} / \mathrm{min}$. First stage thermal desorption temperature (Sample tube temperature) was $260^{\circ} \mathrm{C}$, keeping for $10 \mathrm{~min}$. Cold trap temperature in the first stage thermal desorption process was $-30^{\circ} \mathrm{C}$. From first stage to second stage thermal desorption process heat-up speed was $40^{\circ} \mathrm{C} / \mathrm{s}$. The second stage thermal desorption temperature was $300^{\circ} \mathrm{C}$, maintaining for $5 \mathrm{~min}$. Thermal desorption mode was import diversion, general dry for $10 \mathrm{~min}$. Desorption flow rate 25.00 $\mathrm{ml} / \mathrm{min}$, import diversion flow rate $10.00 \mathrm{ml} / \mathrm{min}$, export diversion flow rate $20.00 \mathrm{ml} / \mathrm{min}$. Chromatographic separation condition that chromatographic column was Elite-5 MS capillary column $(30 \mathrm{~m} \times 0.32 \mathrm{~mm}, 0.252 \mathrm{um})$. Temperature was programmed from $40^{\circ} \mathrm{C}(2 \mathrm{~min}$ hold $)$ to $180^{\circ} \mathrm{C}$ at $6^{\circ} \mathrm{C} / \mathrm{min}(0 \mathrm{~min}$ hold), then increased to $270^{\circ} \mathrm{C}$ at $15^{\circ} \mathrm{C} / \mathrm{min}$ that hold $3 \mathrm{~min}$. Mass spectrometric detection condition was $220^{\circ} \mathrm{C}$ ion source temperature, $250^{\circ} \mathrm{C}$ interface temperature and $29-600$ amu $\mathrm{m} / \mathrm{z}$ scan range. Constituents were identified by peak area normalization method that calculate relative percentage content of each volatile compound from Prunus persica flower in comparison with standard spetral library NIST08 and combined with the retention time and related chemical experience, according to ATD-GC/MS get TIC effective peak.

Sampling method of flower-visiting insect community that sampling of flower-visiting insects which are frequently active in Prunus persica flower were done every 5 - 7 days, from 9:00 to 17:00 on April to June 2016 when 5 trees 
from sunny and shady slope each site, respectively. Photos were taken for insects visiting and foraging nectar and pollen from 3 fixed flower sprays/tree, recording worm access if insects flew away and visited again. Wu (2000), Xue and Zhao (1996) and other taxonomic books were consulted for identifying insect species [29] [30].

One-Way ANOVA analyze different dynamic volatiles, Paired-Sample T-test analysed different slope volatiles with SPSS 17.0.

Flower-visiting insect community parameters were conducted by Patrick richness, abundance and Shannon-Wiener diversity.

Patrick richness is species $R=S$

Abundance is individual $A=N$

Diversity with Shannon-Wiener [31] $H^{\prime}=-\sum P_{i} \ln P_{i} \cdots P_{i}=N_{i} / N$

Correlation analysis VOCs with insect community were conducted by Perason Bivariate correlation analysis volatiles on 29 April with flower-visiting insect richness, abundance and diversity on 25 April, 29 April and 2 May.

\section{Results}

\subsection{Floral Scents}

We detected 20 VOCs emitted from Prunus persica floral branches by ATD-GC/MS, and floral VOCs from sunny slope were more than shady site (17 and 10) (Table 1). On high altitude trees, foral scents from upslope branch are lower than that from downslope of same tree, in accordance with high altitude tree emitted 10 and 15 VOCs from upslope and downslope branches in sunny site, 7 and 8 VOCs from upslope and downslope in shady site. That 6 special floral scents from sunny slope are alpha-dimethyl-benzenemethanol, isoropyl palmitate, ethylbenzene, p-xylene, acetophenone, 3-ethyl-2-methyle-heptane, and 3 special floral scents from shady site are propylene glycol, decanal, hexadecane. Sampled trees from high altitude emit special floral scents are more than low altitude tree, and downslope branches emit special floral scents are more than upslope branches from the same tree except the downslope branches without special scents.

Toluene, hexanal, 2-ethyl-hexanol, dodecane, pentadecane 5 VOCs from Prunus persica floral branches can sampled during 8 temporal quantum, that 1 alcohol, 1 aldehydes, 1 terpene, and 2 hydrocarbons common VOCs emitted from sampled floral branches. The highest pentadecane content emitted from upslope branches on low altitude shady slope during 12:37-12:47, the lowest during 10:39-10:49 (Figure 2). VOCs pentadecane, toluene, 2-ethyl-1-hexanol, hexanal emitted 2 climaxes, while docecane emitted 3 climaxes.

Floral VOCs emitted from floral branches of Prunus persica differently between temporal dynamic sampled trees, the most VOCs 16 constituents emitted from branches of flower during 10:39-11:08, and the lest floral volatiles 8 constituents emitted on the period 11:20-11:47, 12:00-12:24, 12:37-13:01. In addition, 6 common floral scents 2-ethyl-1-hexanol, hexanal, toluene, dodecane, tetradecane, 
Table 1. Floral scents emitted from different branches of Prunus persica in various habitats.

\begin{tabular}{|c|c|c|c|c|c|c|c|c|c|c|c|}
\hline Scent compounds & REV & FOR & $\begin{array}{c}\text { Upslope } \\
\text { branches } \\
\text { on high } \\
\text { altitude } \\
\text { sunny slope }\end{array}$ & $\begin{array}{l}\text { Downslope } \\
\text { branches } \\
\text { on high } \\
\text { altitude } \\
\text { sunny slope }\end{array}$ & $\begin{array}{c}\text { Upslope } \\
\text { branches } \\
\text { on low } \\
\text { altitude } \\
\text { sunny slope }\end{array}$ & $\begin{array}{c}\text { Downslope } \\
\text { branches } \\
\text { on low } \\
\text { altitude } \\
\text { sunny slope }\end{array}$ & $\begin{array}{c}\text { Upslope } \\
\text { branches } \\
\text { on high } \\
\text { altitude } \\
\text { shady slope }\end{array}$ & $\begin{array}{l}\text { Downslope } \\
\text { branches } \\
\text { on high } \\
\text { altitude } \\
\text { shady slope }\end{array}$ & $\begin{array}{c}\text { Upslope } \\
\text { branches } \\
\text { on low } \\
\text { altitude } \\
\text { shady slope }\end{array}$ & $\begin{array}{l}\text { Downslope } \\
\text { branches } \\
\text { on low } \\
\text { altitude } \\
\text { shady slope }\end{array}$ & RT min \\
\hline Total no. & & & 10 & 15 & 8 & 6 & 6 & 7 & 7 & 8 & \\
\hline \multicolumn{12}{|l|}{ Alcohols } \\
\hline Propylene & 811 & 806 & - & 31.255 & 57.968 & - & - & 34.432 & - & - & 1.684 \\
\hline 2-ethyl-1-hexanol & 977 & 946 & 4.990 & 7.374 & 8.324 & 17.699 & 11.616 & 5.743 & 12.352 & 2.464 & 10.432 \\
\hline 2-propyl-1-heptanol & 916 & 890 & - & 1.260 & - & - & - & - & - & - & 11.648 \\
\hline Alpha-dimethyl-benzenemethanol & 951 & 869 & 5.131 & - & - & - & - & - & - & - & 11.918 \\
\hline \multicolumn{12}{|l|}{ Esters } \\
\hline Isoropyl palmitate & 936 & 832 & - & - & 3.365 & - & - & - & - & - & 28.915 \\
\hline Aldehydes & & & & & & & & & & - & - \\
\hline 3-methyl-butanal & 938 & 893 & 3.833 & 3.406 & - & - & - & - & - & - & 3.074 \\
\hline Hexanal & 972 & 952 & 19.559 & 14.340 & 4.875 & 6.852 & 10.503 & 6.117 & 4.782 & 3.182 & 4.805 \\
\hline Heptanal & 972 & 925 & 1.295 & 0.898 & - & - & - & - & - & - & 7.176 \\
\hline Decanal & 949 & 818 & - & - & - & - & - & - & - & 3.352 & 14.844 \\
\hline \multicolumn{12}{|l|}{ Terpenes } \\
\hline Toluene & 991 & 982 & 19.699 & 17.481 & 6.154 & 11.445 & 17.543 & 10.217 & 7.987 & 5.315 & 4.145 \\
\hline Ethylbenzene & 987 & 955 & - & 1.665 & - & - & - & - & - & - & 6.13 \\
\hline P-xylene & 971 & 889 & - & 4.926 & - & - & - & - & - & - & 6.351 \\
\hline Styrene & 965 & 884 & 8.700 & 5.621 & - & - & - & - & - & - & 6.881 \\
\hline Acetophenone & 963 & 893 & - & 2.231 & - & - & - & - & - & - & 11.403 \\
\hline \multicolumn{12}{|l|}{ Hydrocarbons } \\
\hline 3-ethyl-2-methyl-heptane & 906 & 829 & - & 1.017 & - & - & - & - & - & - & 11.528 \\
\hline Dodecane & 983 & 969 & 35.270 & 4.455 & 6.642 & 16.318 & 10.263 & 5.975 & 6.576 & 4.199 & 14.719 \\
\hline Tridecane & 972 & 900 & - & - & - & - & 6.729 & - & 0.145 & - & 17.29 \\
\hline Tetradecane & 965 & 904 & 0.669 & 1.066 & 2.269 & 7.218 & - & 4.845 & 7.622 & 3.352 & 19.246 \\
\hline Pentadecane & 975 & 878 & 0.852 & 3.005 & 10.402 & 40.467 & 43.346 & 32.670 & 60.536 & 48.117 & 21.337 \\
\hline Hexadecane & 972 & 939 & - & - & - & - & - & - & - & 29.879 & 23.247 \\
\hline
\end{tabular}

Notes: RT adopted standard picture with most volatiles, while standard picture without volatiles adopted analysis volatile pictural RT, - unknown.

pentadecane released from trees during 4 temporal quantum (Table 2). LSD analysis indicated that 2-ethyl-1-hexanal, dodecane and tetradecane emitted same content all the temporal quantum, while hexanal, toluene and pentadecane emitted different content some temporal quantum. Floral scents from floral branches of high altitude tree in sunny site are significantly with scents from other kind of trees. 


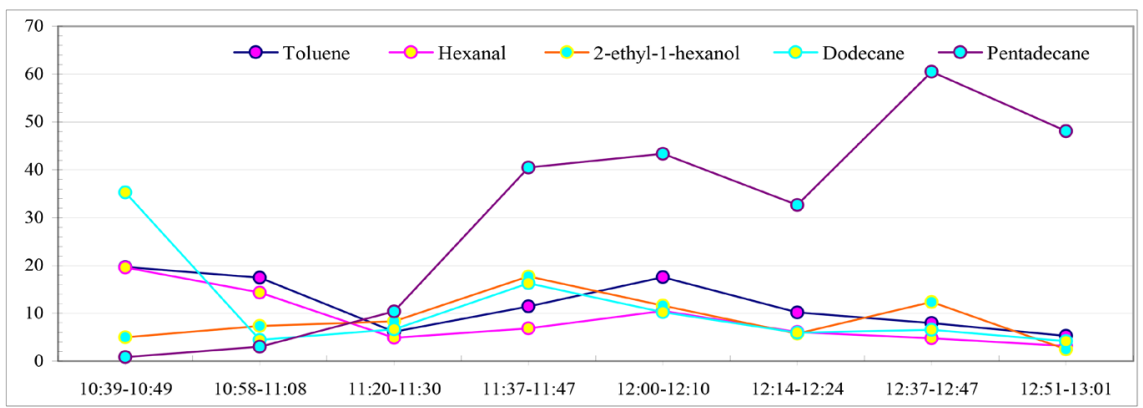

Figure 2. Temporal dynamic of dominant volatiles from floral branches of Prunus persica.

Table 2. LSD analysis floral scents from Prunus persica in different habitats.

\begin{tabular}{|c|c|c|c|c|}
\hline Habitats & $\begin{array}{l}\text { High altitude } \\
\text { tree in sunny } \\
\text { site }\end{array}$ & $\begin{array}{l}\text { Low altitude } \\
\text { tree in sunny } \\
\text { slope }\end{array}$ & $\begin{array}{l}\text { High altitude } \\
\text { tree in shady } \\
\text { slope }\end{array}$ & $\begin{array}{l}\text { Low altitude } \\
\text { tree in shady } \\
\text { slope }\end{array}$ \\
\hline Sample time & 10:39-11:08 & $11: 20-11: 47$ & $12: 00-12: 24$ & $12: 37-13: 01$ \\
\hline \multicolumn{5}{|l|}{ Alcohols } \\
\hline Propylene & $15.62 \pm 22.09 a$ & $28.98 \pm 40.99 a$ & $17.21 \pm 24.35 \mathrm{a}$ & - \\
\hline 2-ethyl-1-hexanol & $6.16 \pm 1.67 \mathrm{a}$ & $13.01 \pm 6.63 a$ & $8.68 \pm 4.15 a$ & $7.41 \pm 6.99 a$ \\
\hline 2-propyl-1-heptanol & $0.63 \pm 0.89$ & - & - & - \\
\hline Alpha-dimethyl-benzenemethanol & $2.56 \pm 3.63$ & - & - & - \\
\hline Isoropyl palmitate & - & $1.68 \pm 2.38$ & - & - \\
\hline \multicolumn{5}{|l|}{ Aldehydes } \\
\hline 3-methyl-butanal & $3.62 \pm 0.30$ & - & - & - \\
\hline Hexanal & $16.95 \pm 3.69 a$ & $5.86 \pm 1.40 \mathrm{~b}$ & $8.31 \pm 3.10 \mathrm{~b}$ & $3.98 \pm 1.13 b$ \\
\hline Heptanal & $1.10 \pm 0.28$ & - & - & - \\
\hline Decanal & - & - & - & $1.75 \pm 2.47$ \\
\hline Terpenes & - & - & - & - \\
\hline Toluene & $18.59 \pm 1.57 \mathrm{a}$ & $8.80 \pm 3.74 \mathrm{~b}$ & $13.88 \pm 5.18 \mathrm{ab}$ & $6.65 \pm 1.89 b$ \\
\hline Ethylbenzene & $0.83 \pm 1.18$ & - & - & - \\
\hline P-xylene & $2.46 \pm 3.48$ & - & - & - \\
\hline Styrene & $7.16 \pm 2.16$ & - & - & - \\
\hline Acetophenone & $1.11 \pm 1.58$ & - & - & - \\
\hline \multicolumn{5}{|l|}{ Hydrocarbons } \\
\hline 3-ethyl-2-methyl-heptane & $0.51 \pm 0.72$ & - & - & - \\
\hline Dodecane & $19.86 \pm 21.79 a$ & $11.48 \pm 6.84 \mathrm{a}$ & $8.12 \pm 3.03 \mathrm{a}$ & $5.39 \pm 1.68 \mathrm{a}$ \\
\hline Tridecane & - & - & $3.37 \pm 4.76 \mathrm{a}$ & $0.07 \pm 0.10 \mathrm{~b}$ \\
\hline Tetradecane & $0.87 \pm 0.28 \mathrm{a}$ & $4.74 \pm 3.50 \mathrm{a}$ & $2.42 \pm 3.43 \mathrm{a}$ & $5.49 \pm 3.02 \mathrm{a}$ \\
\hline Pentadecane & $1.93 \pm 1.52 \mathrm{a}$ & $25.43 \pm 21.26 \mathrm{ab}$ & $38.01 \pm 7.55 b$ & $54.33 \pm 8.78 b$ \\
\hline Hexadecae & - & - & - & $14.94 \pm 12.13$ \\
\hline Floral scent constituent number & 16 & 8 & 8 & 8 \\
\hline
\end{tabular}

Notes: Data were analyzed using LSD test by SPSS 17.0. Mean were value ( \pm sd) from two replications. Same small letters in the same line meant no significant difference at 0.05 levels. 


\subsection{Floral Scents Response to Insect Community}

Insects visit 15 and 4 floral branches of Prunus persica in sunny and shady site, respectively. Most richness, abundance and diversity on sunny or shay slope is similar, while there is difference between SUSD with $n / 2-1$ and $n / 2+1$ (Table 3). Table 4 indicated that floral scent constituent number from sunny slope were significantly negative, and volatile constituent number were remarkably positive correlated with richness, abundance and diversity that insects visiting flowers on sunny or shady slope. Toluene, dodecane were dramatically negative, while tetradecane and pentadecane were significantly positive with SUSR, SUSA, SUSD, SHSR, SHSA and SHSD that FSNS seperated with $\mathrm{n} / 2-1$ or $\mathrm{n} / 2+1$, and FSAS with $\mathrm{n} / 2$.

Floral scents correlated with insect community on sample day, insects only visited Prunus persica flower on shady slope (Table 5 and Table 6). If floral scents from sunny slope were significantly negative correlated with richness, abundance and diversity, while floral scents from shady slope were remarkably positive correlated with those insect community parameters in shady site. 2-ethyl-1-hexanol, tetradecane and pentadecane were significantly positive, while hexanal, toluene, dodecane are dramatically negative correlated with SHSR, SHSA and SHSD separated FSNS with $\mathrm{n} / 2$.

After sampled floral scents day that insects visiting Prunus persica floral branches community index that SUSR response to floral scents correlation with $\mathrm{n} / 2-1$ separated branches is similar to that with $\mathrm{n} / 2+1$, the same occurred at SHSR response to floral scents (Table 7). Moreover, there is difference between SUSA that separated branches with $\mathrm{n} / 2-1$ and $\mathrm{n} / 2+1$, and SHSA, SUSD, SHSD holding the difference. Table 8 indicated that flower-visiting insect SHSA with $\mathrm{n} / 2-1$ separated floral branches and SUSA with $\mathrm{n} / 2+1$ were not correlated with scents from flowers. Floral VOCs and 2-ethyl-1-hexanol from sunny slope were significantly positive correlated with flower-visiting insect community index richness, abundance and diversity on sunny slope, but those scents from shady site were negative correlated with those indexes in sunny and shady sites with

Table 3. Floral scents from Prunus persica and flower-visiting insect community parameters before sampled volatiles day.

\begin{tabular}{ccccccc}
\hline \multicolumn{7}{c}{ Divided insect visiting floral branches from sunny slope with n/2 - 1 and shady slope } \\
with n/2 (FSUS 7/8; FSHS 2/2 )
\end{tabular}

Divided insect visiting floral branches from sunny slope with $\mathrm{n} / 2+1$ and shady slope with n/2 (FSNS 8/7; FSAS 2/2)

Notes: SUVC, SHVC, SUSR, SHSR SUSA, SHSA, SUSD, SHAD, FSUS, FSHS represents volatiles, flower-visiting insect community richness, abundance, diversity, flower branches from sunny and shady slope Prunus persica flower, respectively. The same below. 
Table 4. Floral scents response to flower-visiting insect community parameters before sampled volatiles day.

\begin{tabular}{|c|c|c|c|c|c|c|}
\hline FSUS n/2 - $1 ;$ FSHS n/2 & SUSR & SUSA & SUSD & SHSR & SHSA & SHSD \\
\hline SUVC & $-1.000^{* *}$ & $-1.000^{* *}$ & $-1.000^{\star *}$ & $-1.000^{\star *}$ & $-1.000^{\star *}$ & $-1.000^{* *}$ \\
\hline SHVC & $1.000^{* *}$ & $1.000^{* *}$ & $1.000^{* *}$ & $1.000^{* *}$ & $1.000^{* *}$ & $1.000^{* *}$ \\
\hline SU 2-ethyl-1-hexanol & $1.000^{* *}$ & $1.000^{* *}$ & $1.000^{* *}$ & $1.000^{* *}$ & $1.000^{* *}$ & $1.000^{* *}$ \\
\hline SH 2-ethyl-01-hexanol & $-1.000^{* *}$ & $-1.000^{* *}$ & $-1.000^{* *}$ & $-1.000^{* *}$ & $-1.000^{* *}$ & $-1.000^{* *}$ \\
\hline SU Hexanal & $-1.000^{* *}$ & $-1.000^{* *}$ & $-1.000^{* *}$ & $-1.000^{* *}$ & $-1.000^{* *}$ & $-1.000^{* *}$ \\
\hline SH Hexanal & $-1.000^{* *}$ & $-1.000^{* *}$ & $-1.000^{\star *}$ & $-1.000^{\star *}$ & $-1.000^{\star *}$ & $-1.000^{* *}$ \\
\hline SU Toluene & $-1.000^{* *}$ & $-1.000^{\star *}$ & $-1.000^{* *}$ & $-1.000^{* *}$ & $-1.000^{* *}$ & $-1.000^{* *}$ \\
\hline SH Toluene & $-1.000^{* *}$ & $-1.000^{\star *}$ & $-1.000^{* *}$ & $-1.000^{* *}$ & $-1.000^{\star *}$ & $-1.000^{* *}$ \\
\hline SU Dodecane & $-1.000^{* *}$ & $-1.000^{* *}$ & $-1.000^{* *}$ & $-1.000^{* *}$ & $-1.000^{\star *}$ & $-1.000^{* *}$ \\
\hline SH Dodecane & $-1.000^{\star *}$ & $-1.000^{\star *}$ & $-1.000^{* *}$ & $-1.000^{\star *}$ & $-1.000^{* *}$ & $-1.000^{* *}$ \\
\hline SU Tetradecane & $1.000^{* *}$ & $1.000^{* *}$ & $1.000^{* *}$ & $1.000^{* *}$ & $1.000^{* *}$ & $1.000^{* *}$ \\
\hline SH Tetradecane & $1.000^{* *}$ & $1.000^{* *}$ & $1.000^{* *}$ & $1.000^{* *}$ & $1.000^{* *}$ & $1.000^{* *}$ \\
\hline SU Pentadecane & $1.000^{* *}$ & $1.000^{* *}$ & $1.000^{* *}$ & $1.000^{* *}$ & $1.000^{* *}$ & $1.000^{* *}$ \\
\hline SH Pentadecane & $1.000^{* *}$ & $1.000^{* *}$ & $1.000^{* *}$ & $1.000^{* *}$ & $1.000^{* *}$ & $1.000^{* *}$ \\
\hline SH Pentadecane & $1.000^{\star *}$ & $1.000^{* *}$ & $1.000^{\star *}$ & $1.000^{* *}$ & $1.000^{* *}$ & $1.000^{* *}$ \\
\hline SU Pentadecane & $1.000^{* *}$ & $1.000^{* *}$ & $1.000^{* *}$ & $1.000^{* *}$ & $1.000^{* *}$ & $1.000^{* *}$ \\
\hline SH Tetradecane & $1.000^{\star *}$ & $1.000^{\star *}$ & $1.000^{\star *}$ & $1.000^{* *}$ & $1.000^{* *}$ & $1.000^{* *}$ \\
\hline SU Tetradecane & $1.000^{\star *}$ & $1.000^{\star *}$ & $1.000^{* *}$ & $1.000^{* *}$ & $1.000^{* *}$ & $1.000^{* *}$ \\
\hline SH Dodecane & $-1.000^{\star *}$ & $-1.000^{\star *}$ & $-1.000^{* *}$ & $-1.000^{* *}$ & $-1.000^{\star *}$ & $-1.000^{\star \star}$ \\
\hline SU Dodecane & $-1.000^{\star *}$ & $-1.000^{* *}$ & $-1.000^{* *}$ & $-1.000^{* *}$ & $-1.000^{\star *}$ & $-1.000^{* *}$ \\
\hline SH Toluene & $-1.000^{* *}$ & $-1.000^{* *}$ & $-1.000^{* *}$ & $-1.000^{* *}$ & $-1.000^{\star *}$ & $-1.000^{* *}$ \\
\hline SU Toluene & $-1.000^{* *}$ & $-1.000^{* *}$ & $-1.000^{\star *}$ & $-1.000^{\star *}$ & $-1.000^{* *}$ & $-1.000^{\star *}$ \\
\hline SH Hexanal & $-1.000^{* *}$ & $-1.000^{* *}$ & $-1.000^{* *}$ & $-1.000^{* *}$ & $-1.000^{* *}$ & $-1.000^{* *}$ \\
\hline SU Hexanal & $-1.000^{\star *}$ & $-1.000^{\star *}$ & $-1.000^{\star *}$ & $-1.000^{\star *}$ & $-1.000^{\star *}$ & $-1.000^{\star *}$ \\
\hline SH 2-ethyl-1-hexanol & $-1.000^{* *}$ & $-1.000^{\star *}$ & $-1.000^{* *}$ & $-1.000^{* *}$ & $-1.000^{* *}$ & $-1.000^{* *}$ \\
\hline SU 2-ethyl-1-hexanol & $1.000^{\star *}$ & $1.000^{\star *}$ & $1.000^{\star *}$ & $1.000^{* *}$ & $1.000^{\star \star}$ & $1.000^{\star *}$ \\
\hline SHVC & $1.000^{\star *}$ & $1.000^{\star *}$ & $1.000^{\star *}$ & $1.000^{* *}$ & $1.000^{\star *}$ & $1.000^{\star *}$ \\
\hline SUVC & $-1.000^{\star *}$ & $-1.000^{\star *}$ & $-1.000^{* *}$ & $-1.000^{\star *}$ & $-1.000^{\star *}$ & $-1.000^{\star *}$ \\
\hline FSUS n/2-1; FSHS n/2 & SUSR & SUSA & SUSD & SHSR & SHSA & SHSD \\
\hline
\end{tabular}

Notes: SU, SH represents sunny and shady slope, respectively. ${ }^{* *}$ meant significant correlation at 0.01 level.

Table 5. Flower-visiting insect community parameters on sampled volatiles day.

\begin{tabular}{cccccc}
\hline \multicolumn{5}{c}{ Divided insect visiting floral branches from shady site with n/2 (FSUS 3/3) } \\
\hline SUSR & SHSR & SUSA & SHSA & SUSD & SHSD \\
\hline 0 & $2.50 \pm 0.71$ & 0 & $5.50 \pm 2.12$ & 0 & $0.82 \pm 0.37$ \\
- & - & - & - & - & - \\
\hline SUSR & SHSR & SUSA & SHSA & SUSD & SHSD \\
\hline
\end{tabular}


$\mathrm{n} / 2+1$, respectively. Hexanal, toluene, dodecane was evidently negative, while tetradecane, pentadecane was positive with SUSR, SUSA, SUSD, SHSR, SHSD with $\mathrm{n} / 2-1$. Furthermore, tetradecane, pentadecane was negative, and hexanal, toluene, dodecane was positive with community indexes with $n / 2+1$ separated floral branches.

\section{Discussion}

Our results show that 20 floral scents were emitted from 1 variety Prunus persica branches of flower; that is similar to that 22 constituents produced by 1 variety Hydrocleys marii and 13 constituents produced by 1 variety $H$. nymphoides [24], while 6 floral VOCs were emitted from 6 varieties of Camellia sinensis [32], so Prunus persica flower emitted more VOCs than some plants. Floral scents that were released from sunny slope were more than shady slope, depending

Table 6. Floral scents correlation with flower-visiting insect community parameters on sampled volatiles day.

\begin{tabular}{lcccccc}
\hline \multicolumn{1}{c}{ FSHS n/2 } & SUSR & SUSA & SUSD & SHSR & SHSA & SHSD \\
\hline SUVC & - & - & - & $-1.000^{* *}$ & $-1.000^{* *}$ & $-1.000^{* *}$ \\
SHVC & - & - & - & $1.000^{* *}$ & $1.000^{* *}$ & $1.000^{* *}$ \\
SU 2-ethyl-1-hexanol & - & - & - & $1.000^{* *}$ & $1.000^{* *}$ & $1.000^{* *}$ \\
SH 2-ethyl-1-hexanol & - & - & - & $1.000^{* *}$ & $1.000^{* *}$ & $1.000^{* *}$ \\
SU Hexanal & - & - & - & $-1.000^{* *}$ & $-1.000^{* *}$ & $-1.000^{* *}$ \\
SH Hexanal & - & - & - & $-1.000^{* *}$ & $-1.000^{* *}$ & $-1.000^{* *}$ \\
SU Toluene & - & - & - & $-1.000^{* *}$ & $-1.000^{* *}$ & $-1.000^{* *}$ \\
SH Toluene & - & - & - & $-1.000^{* *}$ & $-1.000^{* *}$ & $-1.000^{* *}$ \\
SU Dodecane & - & - & - & $-1.000^{* *}$ & $-1.000^{* *}$ & $-1.000^{* *}$ \\
SH Dodecane & - & - & - & $-1.000^{* *}$ & $-1.000^{* *}$ & $-1.000^{* *}$ \\
SU Tetradecane & - & - & - & $1.000^{* *}$ & $1.000^{* *}$ & $1.000^{* *}$ \\
SH Tetradecane & - & - & - & $1.000^{* *}$ & $1.000^{* *}$ & $1.000^{* *}$ \\
SU Pentadecane & - & - & - & $1.000^{* *}$ & $1.000^{* *}$ & $1.000^{* *}$ \\
SH Pentadecane & - & - & - & $1.000^{* *}$ & $1.000^{* *}$ & $1.000^{* *}$ \\
\hline
\end{tabular}

Table 7. Flower-visiting insect community parameters after sampled volatiles day.

\begin{tabular}{cccccc}
\hline \multicolumn{5}{c}{ Divided insect visiting floral branches with n/2 - $($ FSUS 1/2; FSHS 3/4 ) } \\
\hline SUSR & SHSR & SUSA & SHSA & SUSD & SHSD \\
\hline $1.50 \pm 0.71$ & $1.50 \pm 0.71$ & $2.00 \pm 1.41$ & $2.00 \pm 0.00$ & $0.32 \pm 0.45$ & $0.35 \pm 0.49$ \\
$1.50 \pm 0.71$ & $1.50 \pm 0.71$ & $5.00 \pm 0.00$ & $5.00 \pm 1.41$ & $0.25 \pm 0.35$ & $0.23 \pm 0.32$ \\
\hline SUSR & SHSR & SUSA & SHSA & SUSD & SHSD \\
\hline \multicolumn{5}{c}{ Divided insect visiting floral branches with n/2 + (FSUS 2/1; FSHS 4/3) } \\
\hline \multicolumn{5}{c}{}
\end{tabular}


Table 8. Floral scents correlation with flower-visiting insect community parameters after sample volatiles day.

\begin{tabular}{|c|c|c|c|c|c|c|}
\hline FSUS or FSHS $n / 2-1$ & SUSR & SUSA & SUSD & SHSR & SHSA & SHSD \\
\hline SUVC & $-1.000^{* *}$ & $-1.000^{* *}$ & $-1.000^{* *}$ & $1.000^{* *}$ & - & $1.000^{* *}$ \\
\hline SHVC & $1.000^{* *}$ & $1.000^{* *}$ & $1.000^{* *}$ & $-1.000^{\star *}$ & - & $-1.000^{* *}$ \\
\hline SU 2-ethyl-1-hexanol & $1.000^{* *}$ & $1.000^{* *}$ & $1.000^{* *}$ & $-1.000^{* *}$ & - & $-1.000^{* *}$ \\
\hline SH 2-ethyl-1-hexanol & $-1.000^{* *}$ & $-1.000^{\star *}$ & $-1.000^{* *}$ & $1.000^{* *}$ & - & $1.000^{* *}$ \\
\hline SU Hexanal & $-1.000^{* *}$ & $-1.000^{* *}$ & $-1.000^{* *}$ & $1.000^{* *}$ & - & $1.000^{* *}$ \\
\hline SH Hexanal & $-1.000^{\star *}$ & $-1.000^{* *}$ & $-1.000^{* *}$ & $1.000^{* *}$ & - & $1.000^{* *}$ \\
\hline SU Toluene & $-1.000^{* *}$ & $-1.000^{* *}$ & $-1.000^{* *}$ & $1.000^{* *}$ & - & $1.000^{* *}$ \\
\hline SH Toluene & $-1.000^{* *}$ & $-1.000^{* *}$ & $-1.000^{* *}$ & $1.000^{* *}$ & - & $1.000^{* *}$ \\
\hline SU Dodecane & $-1.000^{* *}$ & $-1.000^{* *}$ & $-1.000^{* *}$ & $1.000^{* *}$ & - & $1.000^{* *}$ \\
\hline SH Dodecane & $-1.000^{* *}$ & $-1.000^{* *}$ & $-1.000^{* *}$ & $1.000^{* *}$ & - & $1.000^{* *}$ \\
\hline SU Tetradecane & $1.000^{* *}$ & $1.000^{* *}$ & $1.000^{* *}$ & $-1.000^{* *}$ & - & $-1.000^{* *}$ \\
\hline SH Tetradecane & $1.000^{* *}$ & $1.000^{* *}$ & $1.000^{* *}$ & $-1.000^{* *}$ & - & $-1.000^{* *}$ \\
\hline SU Pentadecane & $1.000^{* *}$ & $1.000^{* *}$ & $1.000^{* *}$ & $-1.000^{* *}$ & - & $-1.000^{* *}$ \\
\hline SH Pentadecane & $1.000^{* *}$ & $1.000^{* *}$ & $1.000^{* *}$ & $-1.000^{* *}$ & - & $-1.000^{* *}$ \\
\hline SH Pentadecane & $-1.000^{\star *}$ & - & $-1.000^{\star *}$ & $-1.000^{\star *}$ & $-1.000^{* *}$ & $-1.000^{* *}$ \\
\hline SU Pentadecane & $-1.000^{* *}$ & - & $-1.000^{* *}$ & $-1.000^{\star *}$ & $-1.000^{\star *}$ & $-1.000^{* *}$ \\
\hline SH Tetradecane & $-1.000^{\star *}$ & - & $-1.000^{* *}$ & $-1.000^{* *}$ & $-1.000^{\star *}$ & $-1.000^{* *}$ \\
\hline SU Tetradecane & $-1.000^{\star *}$ & - & $-1.000^{\star *}$ & $-1.000^{* *}$ & $-1.000^{\star *}$ & $-1.000^{* *}$ \\
\hline SH Dodecane & $1.000^{\star *}$ & - & $1.000^{\star *}$ & $1.000^{\star *}$ & $1.000^{\star *}$ & $1.000^{\star \star}$ \\
\hline SU Dodecane & $1.000^{* *}$ & - & $1.000^{* *}$ & $1.000^{\star *}$ & $1.000^{* *}$ & $1.000^{\star *}$ \\
\hline SH Toluene & $1.000^{\star *}$ & - & $1.000^{\star *}$ & $1.000^{* *}$ & $1.000^{* *}$ & $1.000^{\star *}$ \\
\hline SU Toluene & $1.000^{* *}$ & - & $1.000^{\star *}$ & $1.000^{\star *}$ & $1.000^{* *}$ & $1.000^{\star *}$ \\
\hline SH Hexanal & $1.000^{\star *}$ & - & $1.000^{* *}$ & $1.000^{\star *}$ & $1.000^{* *}$ & $1.000^{\star \star}$ \\
\hline SU Hexanal & $1.000^{* *}$ & - & $1.000^{* *}$ & $1.000^{\star *}$ & $1.000^{\star *}$ & $1.000^{\star *}$ \\
\hline SH 2-ethyl-1-hexanol & $1.000^{* *}$ & - & $1.000^{* *}$ & $1.000^{\star *}$ & $1.000^{* *}$ & $1.000^{\star *}$ \\
\hline SU 2-ethyl-1-hexanol & $-1.000^{* *}$ & - & $-1.000^{* *}$ & $-1.000^{* *}$ & $-1.000^{* *}$ & $-1.000^{* *}$ \\
\hline SHVC & $-1.000^{* *}$ & - & $-1.000^{* *}$ & $-1.000^{* *}$ & $-1.000^{* *}$ & $-1.000^{* *}$ \\
\hline SUVC & $1.000^{\star *}$ & - & $1.000^{* *}$ & $1.000^{\star *}$ & $1.000^{* *}$ & $1.000^{\star *}$ \\
\hline FSUS or FSHS $n / 2+1$ & SUSR & SUSA & SUSD & SHSR & SHSA & SHSD \\
\hline
\end{tabular}

on strong sunshine, high temperature, and big humidity affecting floral volatile constituents emitted [33] [34] [35] [36], which testified different chemical substances of fragrance released from Yucca filamentosa on different slopes' geography condition [37]. Moreover, floral volatile constituents of high altitude were more than low altitude that strong sunshine on high altitude promotes flowers emitting floral scents on the same slope [34] [35], and volatiles from 
Ocimum basilicum leaves on high altitude are lower than low altitude in College of Horticulture and Landscape Architecture of Hainan University [18]. Floral scents of 3-methyl-butanal, hexanal were released from Prunus persica floral branches that were emitted from plant leaves defending pests from foraging [38] [39] [40] [41] [42]. We inferred that once insects visit flowers, floral aldehydes were released from Prunus persica, or volatile constituents were released from leaves, when depending on flower and leaf nutrition substance $\mathrm{N}, \mathrm{P}, \mathrm{K}, \mathrm{Ca}, \mathrm{Mg}$, $\mathrm{Fe}, \mathrm{Mn}, \mathrm{Zn}, \mathrm{Na}$ and $\mathrm{Cu}$, which affected fruit [42] [43] [44]. Floral aldehydes were emitted from Prunus persica branches on sunny slope emitted with sunshine. Hydrocarbons such as 3-ethyl-2-methyl-heptane, dodecane, tridecane, tetradecane, pentadecane and hexadecane were released from Prunus persica branches, and hydrocarbons produce a pleasant smell [45] [46], so 6 floral hydrocarbons from Prunus persica attracted insects visiting. Dissimilarity with ATD-GC/MS analysed dominant volatile constituents from Agiceras comiculatum terpenes with poison [47], and dissimilarity with floral volatiles from Osmanthus fragrans ketones and alcohols [11] [19] [48].

Floral VOCs were differently emitted from Prunus persica on temporal quantum, and difference VOCs of flower were released from Sorbaria kirilowii on different seasons with TCT-GC/MS analysis [49], while floral scents were produced from Lilium brownii on open period, semi-open, blooming period and recession period [14], but VOCs were emitted from Rosa rugosa and Sorbaria kirilowii with diurnal rhythm variation [50] [51]. Most floral scents of 16 constituents were emitted from Prunus persica on 10:39-11:08, while same 8 constituents were released from trees on other temporal quanta, which is related with sunshine and temperature [34] [52], and flower quantity affected volatiles release, different floral volatiles from Agiceras comiculatum branches without flower, with flower and with fruit [47]. Floral VOCs from Prunus persica before 12:00 are higher than those after 12:00; special floral scents were emitted from both temporal quanta, while floral terpenes and esters were emitted most from Arabian jasmine florescence [53]. Floral hexanal was released in all the sampling time and content from Prunus persica is significantly highest during 10:30-11:30, while hexanal was released from Sorbaria kirilowii in all the sampling time [9]. Pink flowers from Prunus persica and white flowers from Sorbaria kirilowii absorb and reflect different wavelength of light, which release a different amount of hexanal, and flower color has the characteristics of adaptive environment [54]. LSD analysis indicated that there was significantly same floral scent dynamic difference of some constituents, same floral volatile constituents emitted different contents from Sorbaria kirilowii during 7:00-19:00 [9], and same floral scent constituents emitted different contents from Rosa rugosa one day [51]. There was same floral volatile significant difference of common constituents among some temporal quantum, while VOCs were emitted significant difference from Sorbaria kiriowii on different temporal quanta one day, and floral scents were differently released from Lilium brownii on different period [9] [14]. 
Floral scent correlation with insect community that floral VOCs emission responded to insects visiting Prunus persica flower branches and floral VOCs total content and relative content of each component response to Mylabris forage [41]. Methyl benzenecarboxylate emitted from pollinated Pentunia flower with certain rules [55], while more volatiles released from the plant that foraged by insects to attract enemy insects, and volatile emission correlated with foraging insect population [56]. Flavoring substance VOCs emission from Cirsium flowers increased with flower-visiting insect community index ascend [57], while VOCs emitted from needles correlated with Dendrolimus punctatus burst frequently [58], furthermore, fragrance floral VOCs emitted from Heterobathmiina pollinated flowers are higher than that Aves pollinated flowers [59]. We first founded that there were different correlation between floral scents and insect community visited Prunus persica floral branches before sampled day, that odd branch number from sunny slope and even branches from shady slope separated with $n / 2-1$ or $n / 2+1$, and $n / 2$, but the floral scent constituents is negative correlated with flower-visiting insect community richness, abundance and diversity on the sunny slope, and positive correlated with those indexes adopted whichever separated method, so could adopted some floral scents that positive correlated with flower-visiting insects on sunny slope to attract insects pollinating [24] [60]. We firstly inferred that floral scents response to flower-visiting insect community depending on the tree branch number and even branches distributed slopes, because 2-ethy-1-hexanal, toluene, dodecane are dramatically negative, while tetradecane and pentadecane are significantly positive with SUSR, SUSA, SUSD, SHSR, SHSA and SHSD that FSUS separated with n/2 - 1 or n/2 + 1 , and FSHS with $\mathrm{n} / 2$. We concluded that 2-ethyl-1-hexanol from sunny and shady slope could be used to attract insects visiting flower of Prunus persica, in that insects only visiting floral branches on shady slope that holding odd floral branches, and 2-ethyl-1-hexanol from sunny and shady slope were positive correlated with SHSR, SHSA and SHSD on sampled volatiles day [24]. But, after sampled volatiles day floral scent constituents and 2-ethyl-1-hexanol on sunny slope were positive correlated with SUSR, SUSA, SUSD, and negative correlated with SHSR and SHSD, that separated floral branches with $n / 2-1$. So 2-methyl-1-Hexanol from sunny and shady slope could used to lure insects to pollinate on separate slopes, just as flora scents from one kind of plant attract insects pollinating [55] [58] [59]. While no quantitative insects visiting floral branches of Prunus persica that disadvantage sunshine and temperature climate condition on shady slope [61], and once insects visit floral branches, volatile constituents and quantity emitted possibly from Prunus persica to attract insects visiting to pollinate or inhibit insects foraging flowers, so floral scents were significantly related with flower-visiting insect community indexes [56]. Advantage sunshine and temperature climate condition on sunny slope [61] distributed more flower-visiting insect species that pollinated easily. In addition, flower structural characteristics, the amount and composition of nectar affect flower-visiting 
insect species [62] [63]. When insects visiting, less floral volatile constituents and contents emitted from Prunus persica branches to inhibit insects from visiting, and secondary metabolic substances emitted from Prunus persica branches to inhibit insects from visiting [8].

Floral branches of Prunus persica pollinated are in a physiological and biochemical state that is to pollinate. Punus persica in Wan Bailin Ecological Garden healthily grow and fruit need insect visiting and emitters attract and inhibitory floral scents, we first definite the 20 floral scents, dominant and special fragrance of Prunus persica that which constituents are attractive could be specific to lure insects pollinating in the future, and perhaps that 2-ethyl-1-hexanol is likely to attract insects, mostly. We first presented that floral scents emission correlated with floral branches and hold different response to flower-visiting insect community indexes separated floral branches with even or odd methods, that prune Prunus persica tree leaving branches odd on sunny slope and even on shady slope to promote those tree growing and fruiting, and get high economic benefit.

\section{Acknowledgements}

This study was financially supported from "Shanxi Forestry Vocational Technical College Scientific Research and Teaching Reform Fund in China (201502)" and "Shanxi Scholarship Council of China 2016 Year Provincial Fund in China". The authors would like to thank Dongcheng Mao and Zhejuan Wang for Volatiles sampled in the Garden.

\section{References}

[1] Leonhardt, S.D., Baumann, A.M., Wallace, H.M., Brooks, P. and Schmitt, T. (2014) The Chemistry of an Unusual Seed Dispersal Mutualism: Bees Use a Complex Set of Olfactory Cues to Find Their Partner. Animal Behaviour, 98, 41-51. https://doi.org/10.1016/j.anbehav.2014.09.024

[2] Zito, P., Doetterl, S. and Sajeva, M. (2015) Floral Volatiles in a Sapromyiophilous Plant and Their Importance in Attracting House Fly Pollinators. Journal of Chemical Ecology: Official Journal of the International Society of Chemical Ecology, 41, 340-349. https://doi.org/10.1007/s10886-015-0568-8

[3] Danny, K. and Ian, T.B. (2006) Making Sense of Nectar Scents: The Effects of Nectar Secondary Metabolites on Floral Visitors of Nicotiana attenuata. The Plant Journal, 49, 840-854.

[4] Pat, G.W., Clive, V.N., Nigel, E.R., Graham, N.S., Jonathan, G.P., Kate, H., Philip, S., Lynn, M., Simon, G.P. and Jeffe, T.K. (2009) Floral Volatiles Controlling ant Behavior. Functional Ecology, 23, 888-900. https://doi.org/10.1111/j.1365-2435.2009.01632.x

[5] Chen, Y.L., Fang, L.N. and Wu, W.Z. (2010) The Effect of Infochemicals on Selection Behavior of Wiebesia pumilae (Hymenoptera:Chalcidoidea:Agaonidae). Acta Ecologica Sinica, 30, 2949-2957.

[6] Cesar, R.S., Leonardo, P., Andrés, Q. and Rufus, I. (2011) Variation in Highbush Blueberry Floral Volatile Profiles as a Function of Pollination Status, Cultivar, Time of Day and Flower Part: Implications for Flower Visitation by Bees. Annals of Bo- 
tany, 107, 1377-1390. https://doi.org/10.1093/aob/mcr077

[7] Heil, M. (2008) Indirect Defence Recent Developments and Open Questions. Progress in Botany, 69, 360-395. https://doi.org/10.1007/978-3-540-72954-9_15

[8] Danesi, F., Saha, S., Kroon, P.A., Glietić, M., Konić-Ristić, A., D’Antuono, L.F. and Bordoni, A. (2013) Bioactive-Rich Sideritis scardica Tea (Mountain Tea) Is as Potent as Camellia sinensis Tea at Inducing Cellular Antioxidant Defences and Preventing Oxidative Stress. Journal of the Science of Food and Agriculture, 93, 3558-3564. https://doi.org/10.1002/jsfa.6214

[9] Li, H.D., Gao, Y. and Jin, Y.J. (2004) The Daily Dynamic Variances of the VOCS Releasing from Flower of Siberia kirilow II (Regel) Maxim. Journal of Inner Mongolia Agriculture University (Natural Science Edition), 25, 54-59.

[10] Li, Y.Y. (2012) The Main Composition and Affecting Factors of Aroma Volatiles in Flowers. Northern Horticulture, No. 6, 184-187.

[11] Wu, D., Luo, S.Q., Yang, Z.N., Ma, J. and Yu, Z.W. (2014) Characteristics of Volatile Phytochemical Components of Different Organs of Osmanthus fragrans var. latifolius. Southwest China Journal of Agricultural Sciences, 27, 1243-1247.

[12] Salvatore, C., Silvia, F., Maria, L., Giovanii, S., Judith, T. and Florian, P.S. (2015) Herbivory Increases Fruit Set in Silene latifolia: A Consequence of Induced Pollinator-Attracting Floral Volatiles? Journal of Chemical Ecology, 41, 622-630. https://doi.org/10.1007/s10886-015-0597-3

[13] Cheng, M., Zhang, Y.X., Feng, X.F., Yang, L.J., Yang, L.X., Ge, X.G., Wang, H.J. and Hu, S.L. (2012) GC-MS Analysis on N-hexane Extractive of Fresh Flos Paeoniae Rubra and Flos Paeoniae Alba. Chinese Wild Plant Resources, 31, 17-19.

[14] Zhang, H.X., Hu, Z.H., Leng, S.P., Wang, W.H., Xu, F. and Zhao, J. (2012) Dynamic Changes of Aroma Components of 'White Heaven' Lily during Different Florescences. Northern Horticulture, No. 16, 68-71

[15] Zhang, J., Zhou, X.T., Hu, L.P. and Zhou, Z.R. (2013) SPME-GC-MS Measurement of Volatile in Different Peony Varieties. Journal of Northwest Forestry University, 28, 136-143.

[16] Joshi, R., Poonam and Gulati, A. (2011) Biochemical Attributes of Tea Flowers (Camellia sinensis) at Different Developmental Stages in the Kangra Region of India. Scientia Horticulturae, 130, 266-274. https://doi.org/10.1016/j.scienta.2011.06.007

[17] Shen, L.Q. and Lei, F.H. (2010) Analysis of Volatile Components in Pitaya Flower by Headspace Solid Phase Microextraction-GC/MS. Food Science, 31, 315-317.

[18] Song, S.Q., Gu, M., Chen, F.P. and Xu, B.Q. (2008) Analysis of Volatile Compounds from Flowers and Leaves of Ocimum basilicum L. with Solid Phase Microextraction by GC/MS. Journal of Chinese Mass Spectrometry Society, 29, 110-114.

[19] Jin, H.X., Zheng, H., Jin, Y.J., Chen, J.Y. and Wang Y. (2006) Research on Major Volatile Components of 4 Osmanthus fragrance Cultivars in Hangzhou Manlong Guiyu Park. Forest Research, 19, 612-615.

[20] Kang, W.Y. and Xu, Q.T. (2008) Rapid Determination of Volatile Constituents from the Buds and Flowers of Prunus persica (L.) Batsch F. Duplex Rehd. 2008 2nd International Conference on Bioinformatics and Biomedical Engineering, Shanghai, 16-18 May 2008, 1024-1027. https://doi.org/10.1109/ICBBE.2008.251

[21] Montero-Prado, P., Bentayeb, K. and Nerín, C. (2013) Pattern Recognition of Peach Cultivars (Prunus persica L.) from Their Volatile Components. Food Chemistry, 138, 724-731. https://doi.org/10.1016/j.foodchem.2012.10.145 
[22] Andrews, E.S., Theis, N. and Adler, L.S. (2007) Pollinator and Herbivore Attraction to Cucurbita Floral Volatiles. Journal of Chemical Ecology, 33, 1682-1691. https://doi.org/10.1007/s10886-007-9337-7

[23] Borges, R.M., Bessière, J.M. and Ranganathan, Y. (2013) Diel Variation in Fig Volatiles across Syconium Development: Making Sense of Scents. Journal of Chemical Ecology, 39, 630-642. https://doi.org/10.1007/s10886-013-0280-5

[24] Carvalho, A.T., Dötterl, S. and Schelindwein, C. (2014) An Aromatic Volatile Attracts Oligolectic Bee Pollinators in an Interdependent Bee-Plant Relationship. Journal of Chemical Ecology, 40, 1126-1134. https://doi.org/10.1007/s10886-014-0510-5

[25] Milet-Pinheiro, P., Ayasse, M., Dobson, H.E.M., Schlindwein, C., Francke, W. and Dötterl, S. (2013) The Chemical Basis of Host-Plant Recognition in a Specialized Bee Pollinator. Journal of Chemical Ecology, 39, 1347-1360. https://doi.org/10.1007/s10886-013-0363-3

[26] Bruinsma, M., Lucas-Barbosa, D., ten Broeke, C.J., van Dam, N.M., van Beek, T.A., Dicke, M. and van Loon, J.J. (2014) Folivory Affects Composition of Nectar, Floral Odor and Modifies Pollinator Behavior. Journal of Chemical Ecology, 40, 39-49. https://doi.org/10.1007/s10886-013-0369-x

[27] Burdon, R.C.F., Raguso, R.A., Kessler, A. and Parachnowitsch, A.L. (2015) Spatiotemperal Floral Scent Variation of Penstemon digitalis. Journal of Chemical Ecology, 41, 641-650. https://doi.org/10.1007/s10886-015-0599-1

[28] Wright, G.A. and Schiestl, F.P. (2009) The Evolution of Floral Scent: The Influence of Olfactory Learning by Insect Pollinators on the Honest Signalling of Floral Rewards. Functional Ecology, 23, 841-851. https://doi.org/10.1111/j.1365-2435.2009.01627.x

[29] Wu, Y.R. (2000) Fauna Sinica Insecta (Vol. 20), Hymenoptera, Melittidae Apidae. Science Press, Beijing.

[30] Xue, W.Q. and Zhao, J.M. (1996) Flies of China. Liaoning Science and Technology Publishing House, Shenyan.

[31] Feng, L.C., Meng, Q.F. and Gao, W.T. (2015) Diversity and Behavior of Flower Visitors Insects of Malus komarovii in the Southwest Slope of Changbai Mountain. Guangdong Agricultural Sciences, 42, 147-152.

[32] Zeng, L., Fu, L.Y., Luo, L.Y., Ma, M.J. and Li, S. (2015) Principal Component and Cluster Analyses of Volatile Components in Tea Flowers from Different Cultivars at Different Stages of Bloom. Food Science, 36, 88-93.

[33] Chai, Q. and Gao, B. (2003) Review on Action Mechanism Affecting Factors and Applied Potential of Allelopathy. Acta Botanica Boreali-Occidentalia Sinica, 23, 509-515.

[34] Lu, S.P. and Sui, X.X. (2006) Biological Functions of Secondary Metabolism of Medicinal Plants and Influences of Ecological Environment. Natural Product Research and Development, 18, 1027-1032.

[35] Que, X.X., Huang, Y. and Bai, X.D. (2013) Correlation between Flowering Phase of Osmanthus fragrans and Climate Factors in Guilin. Journal of Southern Agriculture, 44, 1831-1834.

[36] Reig, G., Iglesias, I., Miranda, C., Gatius, F. and Alegrea, S. (2013) How Does Simulated Frost Treatment Affect Peach [Prunus persica (L.)] Flowers of Different Cultivars from Worldwide Breeding Programmes? Scientia Horticulturae, 160, 70-77. https://doi.org/10.1016/j.scienta.2013.05.021 
[37] Svensson, G.P., Hickman, M.O., Bartram, S., Boland, W., Pellmyr, O. and Raguso, R.A. (2005) Chemistry and Geographic Variation of Floral Scent in Yucca filamentosa (Agavaceae). American Journal of Botany, 92, 1624-1631. https://doi.org/10.3732/ajb.92.10.1624

[38] Zhang, X.B., Wang, J., Xie, L. and Zhang, Y.D. (2013) Comparative Analysis on Volatile Compounds of Flowers, Leaves and Stems of Basil. Chinese Journal of Tropical Crops, 4, 1182-1187.

[39] Hidebrand, D.F., Brown, G.C., Jackson, D.M. and Hamilton-Kemp, T.R. (1993) Effects of Some Leaf-Emitted Volatile Compounds on Aphid Population Increase. Journal of Chemical Ecology, 19, 1875-1887. http://doi.org/10.1007/BF00983793

[40] Guo, H., Hou, D.Y., Hui, R.H. and Li, T.C. (2006) Analysis of Volatile Constituents of Flowers of L. Sativa var. angustana Irish by GC/MS. Journal of Chinese Mass Specreometry Society, 27, 113-116.

[41] Cheng, M., Feng, X.F., Yan, H., Yang, L.J., Yang, L.X., Zhang, Y.X., Ge, X.G., Ji, L. and Hu, S.L. (2010) GC-MS Analysis on Volatile Compounds of Peaonia lactiflora Petals after Eaten by Mylabris speoiosa. Chinese Journal of Experiment Traditional Formulae, 16, 30-33.

[42] Sanz, M. and Montañés, L. (1995) Flower Analysis as a New Approach to Diagnosing the Nutritional Status of the Peach Tree. Journal of Plant Nutrition, 18, 1667-1675. https://doi.org/10.1080/01904169509365012

[43] Zarrouk, O., Gogorcena, Y., Gómez-Aparisi, J., Betrán, J.A. and Moreno, M.A. (2005) Influence of Almond $\times$ Peach Hybrids Rootstocks on Flower and Leaf Mineral Concentration, Yield and Vigour of Two Peach Cultivars. Scientia Horticulturae, 106, 502-514. https://doi.org/10.1016/j.scienta.2005.04.011

[44] Yamane, H., Ooka, T., Jotatsu, H., Sasaki, R. and Tao, R. (2011) Expression Analysis of PpDAM5 and PpDAM6 during Flower Bud Development in Peach (Prunus persica). Scientia Horticulturae, 129, 844-848. https://doi.org/10.1016/j.scienta.2011.05.013

[45] Yang, J.Y. and Meng, H.J. (2007) Research Progress of Durian and Human Health. China Fruit News, 24, 29-30.

[46] Zhang, H., Zheng, H., Feng, Y., Yu, L.S. and Zhang, R.G. (2008) Analysis of Volatile Components of "Jinzhen" Durian Fruit by TCT-GC/MS. Food Science, 29, 517-519.

[47] Yao, Y.L., Zheng, H., Lu, X.F., Li, K., Zhong, J.C., Song, G.B. and Chen, D.L. (2015) Composition of Natural Volatiles from Aegiceras comiculatum (L.) Blanco and Perspective on Its Application. Journal of Anhui Agricultural Sciences, 43, 177-178, 181.

[48] Chen, H.X. and Wang, C.Z. (2012) Analysis of Essential Oil Compounds from Different Species Osmanthus fragrans lour. by Gas Chromatography-Mass Spectrometry. Biomass Chemical Engineering, 46, 38-41.

[49] Zheng, H., Jin, Y.J., Zhou, J.X. and Li, W.B. (2003) A Preliminary Study on Human Brain Waves Influenced by Volatiles Released from Living Sorbaria kirilowii (Regel) Maxim. in Different Seasons. Forest Research, 16, 328-334.

[50] Li, H.D., Gao, Y. and Jin, Y.J. (2004) The Daily Dynamic Variances of the VOCS Releasing from Flower of Siberia kirilow II (Regel) Maxim. Journal of Inner Mongolia Agriculture University (Natural Science Edition), 25, 54-59.

[51] Hendel-Rahmanim, K., Masci, T, Vainstein, A. and Weiss, D. (2007) Diurnal Regulation of Scent Emission in Rose Flower. Planta, 226, 1491-1499. https://doi.org/10.1007/s00425-007-0582-3

[52] Raguso, R.A. (2008) Wake Up and Smell the Roses: The Ecology and Evolution of 
Floral Scent. Annual Review of Ecology, Evolution, and Systematics, 39, 549-569. https://doi.org/10.1146/annurev.ecolsys.38.091206.095601

[53] Zhang, Q., Gao, X.X. and Wang, P. (2015) Volatile Components Analysis of Five Flower Phases of Jasminum sambac cv. Bifoliatum by GC-MS. Chinese Journal of Tropical Crops, 36, 792-797.

[54] Newman, E., Anderson, B. and Johnson, S.D. (2012) Flower Colour Adaptation in a Mimetic Orchid. Proceedings of the Royal Society B: Biological Sciences, 279, 2309-2313. https://doi.org/10.1098/rspb.2011.2375

[55] Negre, F., Kish, C.M., Boatright, J., Underwood, B., Shibuya, K., Wagner, C., Clark, D.G. and Dudareva, N. (2003) Regulation of Methylbenzoate Emission after Pollination in Snapdragon and Petunia flowers. The Plant Cell, 15, 2992-3006.

https://doi.org/10.1105/tpc.016766

[56] Clavijo Mccormick, A., Irmisch, S., Reinecke, A., Boeckler, G.A., Veit, D., Reichelt, M., Hansson, B.S., Gershenzon, J., Köllner, T.G. and Unsicker, S.B. (2014) Herbivore-Induced Volatile Emission in Black Poplar: Regulation and Role in Attracting Herbivore Enemies. Plant, Cell \& Environment, 37, 1909-1923.

https://doi.org/10.1111/pce.12287

[57] Theis, N., Lerdau, M. and Raguso, R.A. (2007) The Challenge of Attracting Pollinators while Evading Floral Herbivores: Patterns of Fragrance Emission in Cirsium arvense and Cirsium repandum (Asteraceae). International Journal of Plant Sciences, 168, 587-601. https://doi.org/10.1086/513481

[58] Zhang, S.F., Zhang, Z., Kong, X.B., Wang, H.B., Cai, B.X. and Tao, Y. (2015) Volatile Characters of Masson Pine Needles from Dendrolimus punctatus Frequent Outbreak Area, Occasional Outbreak Area, and Non-Outbreak Area. Scientia Silvae Sinicae, 51, 170-174.

[59] Van der Niet, T., Pirie, M.D., Shuttleworth, A., Johnson, S.D. and Midgley, J.J. (2014) Do Pollinator Distributions Underlie the Evolution of Pollination Ecotypes in the Cape Shrub Erica plukenetii? Annals of Botany, 113, 301-316. https://doi.org/10.1093/aob/mct193

[60] Knudsen, J.T., Eriksson, R., Gershenzon, J. and Ståhl, B. (2006) Diversity and Distribution of Floral Scent. The Botanical Review, 72, 1. https://doi.org/10.1663/0006-8101(2006)72[1:DADOFS]2.0.CO;2

[61] Wang, X.H., Ma, L.Z., Ma, X.H., Chen, L.Y. and Zhou, Z.C. (2011) Growth and Wood Basic Density of Schima superba Plantation. Scientia Silvae Sinicae, 47, 138-144.

[62] Van der Niet, T., Jürgens, A. and Johnson, S.D. (2010) Pollinators, Floral Morphology and Scent Chemistry in the Southern African Orchid Genus Schizochilus. South African Journal of Botany, 76, 726-738. https://doi.org/10.1016/j.sajb.2010.07.004

[63] Steiner, K.E., Kaiser, R. and Dotterl, S. (2011) Strong Phylogenetic Effects on Floral Scent Variation of Oil-Secreting Orchids in South Africa. American Journal of Botany, 98, 1663-1679. https://doi.org/10.3732/ajb.1100141 